\title{
Wireless Sensor Network Route Optimization Based on Improved Ant Colony-Genetic Algorithm
}

\author{
http://dx.doi.org/10.3991/ijoe.v11i9.5057 \\ Yongfeng Cui* ${ }^{1}$, Wei Liu ${ }^{1}$, Zhongyuan Zhao ${ }^{1,2}$ \\ ${ }^{1}$ Zhoukou Normal University, Zhoukou, Henan, China \\ ${ }^{2}$ Henan University of Technology, Zhengzhou, Henan China
}

\begin{abstract}
The objective of this paper is focuses on route optimization, for a given wireless sensor network. We detail the significance of route optimization problem and the corresponding mathematical model. After analyzing the complex multi-objective optimization problem, Ant Colony Optimization (ACO) algorithm was introduced to search the best route. Inspired by Genetic Algorithm (GA), we embed two operations into ACO to refine it. First, every ant after achieving sink will be regarded as an individual such as that in GA. The crossover operation will be applied and then, the generated new ants will replace the weaker parents. Second, we designed a mutation operation for ants selecting next nodes to visit. Experimental results demonstrate that the proposed combination algorithm has significant enhancements than both GA and ACO. The lifetime of WSN can be extended and the coverage speed can be accelerated.
\end{abstract}

Index Terms-Ant Colony, GA, Route Optimization, WSN.

\section{INTRODUCTION}

Wireless Sensor Network (WSN) is a synthesized problem which combining embedding technology, sensor communication technology and distributed information processing technology [1]. Commonly, a WSN is constructed by a certain sensor with energies and selforganized. Currently, WSN was widely used in biomedical, environmental monitoring, military defense and other fields, which has a very broad application prospect [2].

A complete wireless sensor network system comprises a distributed group of sensor nodes, sink and Internet [3]. The working theory is briefly detailed as follows: 1 . Sensor nodes using the data acquisition module collected data. 2. The data was transferred to sink using forwarding module according to the routing protocol. 3. Sink node connects the Internet via transceiver stations. 4. Complete data collection, processing and analysis tasks. The illustration of a typical WSN was showed in Fig.1.

Compared with traditional wireless networks, the energy of each sensor node is limited and the node can't be replaced. Thus, reducing energy consumption is the core issue of WSN [4]. The largest consumption in the operation of WSN is information transmissions. Hence, route optimization problem is the most important task to maximize the life cycle of WSN [5]. Because of the limited energy of sensor nodes, route optimization design not only considers the length of the data transmission path, but also considers saving energy and network energy balance and other issues. WSN route optimization not only concerned about the path length, are more concerned about saving energy and balance energy consumption of the whole network [6][7].

So, wireless sensor network route optimization is a typical multi-objective optimization problem. The objective of WSN route optimization is to find and build energyefficient, reliable routes from sensor nodes to the receiving node (Sink) for data transmission to maximize the life cycle of WSN [8][9].

In summary, the route optimization of wireless sensor network is to find the shortest data transmission path between a source node and sink node with least energy consumption and the longest life cycle, which is a complex multi-objective optimization problem. Calculation times of traditional optimization algorithms are long and this doesn't satisfy the requirements of real-time communication. Traditional methods for solving WSN route optimizations are based on Dynamic Programming (DP) such as Dijkstra [21] and Floyd-Warshall [22]. Although DP can obtain the optimal solution, the calculation consumption is very large. Another reason is that, the mathematical model of DP is difficult to link to the complex route optimization problem. Recently, optimization algorithms based on artificial intelligence shows excellent performances on solving combination optimization problems such as Genetic Algorithm (GA) [10], Ant Colony Optimization (ACO) [9], Simulate Annealing (SA) algorithm [11] and Particle Swarm Optimization (PSO) [12]. They have respective advantages, but their disadvantages are also apparent. Using a single algorithm is difficult to find the optimal WSN route. In this paper, we combine GA and ACO then design two operations to form a superior optimization algorithm, which is a powerful wireless sensor network path optimization tool with less time consumption and higher precision.

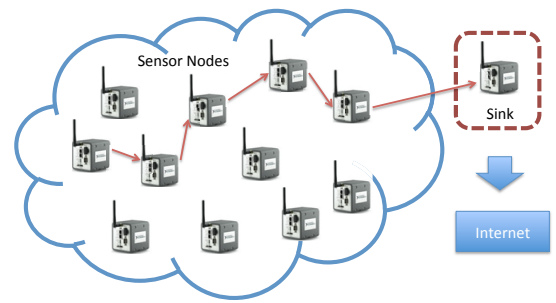

Figure 1. An illustration to exhibit the structure of WSN. The red lines formed a transmission route from sensor nodes to Sink and then the information after aggregation is emitted to internet. 
The rest of this paper is organized as follows: Section 2 describes the mathematical model of WSN path optimization briefly. Ant Colony algorithm and Genetic algorithm are introduced in Section 3 and the combination algorithm was proposed consequently. Section 4 exhibits the experimental results of our improved model and gives the comparison results of the state-of-arts algorithms. Conclusion and further studies are discussed in Section 5.

\section{MAthematical Model of ROUTE Optimization PROBLEM IN WSN}

In order to describe the route optimization problem explicitly, we briefly give the corresponding mathematical model. Wireless sensor networks make the following assumptions:

1). Each sensor node has a unique ID number.

2). Nodes of WSN are distributed in two-dimensional space; each sensor node has the same performance on transmission and communication.

3). The initial energy of each sensor node is equal; the location is fixed and can't to be moved.

In the operation of wireless sensor networks, the node that has collected monitoring data is regarded as the source node. Then, the data gather through multi-hop to sink node (the target node). We can utilize an undirected weighted graph $G=\langle V, E>$ to describe a WSN abstractly. $V$ denotes the wireless sensor network nodes set and $E$ represents communication links set as follows:

$$
\begin{gathered}
V=\left\{V_{\text {head }}, v_{1}, v_{2}, \ldots, v_{n}\right\} \\
) \\
E=\left\{e_{1}, e_{2}, \ldots, e_{n}\right\}
\end{gathered}
$$

Wherein, $V_{\text {head }}$ denotes the cluster head of $G, v_{i}$ denotes the other perception nodes.

Let $\lambda_{0}$ denotes the effective transmission distance in $V$, we have:

$$
\begin{gathered}
E=\left\{e_{i} \mid D\left(v_{j}, v_{k}\right) \leq \lambda_{0}\right\} \\
v_{j}, v_{k} \in V
\end{gathered}
$$

The connection matrix $L$ can be calculated as follow:

$$
L_{i j}=L_{j i}=\left\{\begin{array}{lc}
1, & \text { if valid } \\
0, & \text { else }
\end{array}\right.
$$

The objective function is defined as follow:

$$
F=\min \left(\sum_{i=1}^{N-1} L_{i, i+1} D_{i, i+1}+\sum_{i=1}^{N-1} L_{i, i+1} E_{i}+\sum_{i=2}^{N} L_{i-1, i} e_{i}\right)
$$

Wherein, $N$ denotes the number of the nodes in one route; this is not a fixed number. $D_{i, i+1}$ denotes the distance between the $i$-th and the $i+1$-th nodes in the route. $E_{i}$ denotes the energy consumption of the $i$-th node in the route for emission data and $e_{i}$ denotes the energy consumption for receiving data.

The goal of our task optimizing the route of WSN is to find the best data transmission path between a source node and the target point.

\section{IMPROVED COMBINATION ALGORITHM FOR WSN ROUTE OPTIMIZATION}

Ant colony optimization algorithm finds the optimal solution by accumulating the pheromone, which is an excellent algorithm with global parallel and distributed search capabilities [13][14]. But the initial pheromone is merely and leading to a low convergence speed; thus reducing the overall performance of the algorithm [15][16]. GA has global search capability; its search speed is fast and it has an extraordinary performance for solving large-scale optimization problems [17]. However, GA can't feedback the pheromone to itself like ACO, when the algorithm is calculated after a certain iterations, GA will produce numerous redundant information thus the performance declined [18]. In this section, a novel combination optimization algorithm will emerge after analysis the pros and cons of the two algorithms.

\section{A. Ant Colony Optimization Algorithm}

ACO is a swarm intelligence algorithm devised by simulating the schematic of ants foraging. Ants can leave a substance called pheromone on its path in the foraging process and it also can perceive the strength of this material in the feeding process. By exploiting the degree of pheromone, ants can guide their direction of action, they always moving toward the path with more pheromones. The implied optimal path will have more and more pheromone after certain iterations. This is a virtuous cycle and the optimal implied path will emerge from a large number of ants' feedback.

The searching processing of artificial ants includes two behaviors:

1) Memory behavior: the path that passed by an ant will no longer select by it again. Hence, a taboo table is necessary for ACO to guarantee this rule.

2) Communication behavior: ants use pheromones to communicate with each other. The path that chose by ants will be added some pheromone and willhave more attractive for other ants. An ant in front of a fork will observe the pheromone degrees of the branches and they are more likely to select the branch with the most pheromone.

An illustration of ACO iteration processing is showed in Fig.2. The up figure shows that the initial status of the colony and the bottom is the status after a certain of iteration. Ants are more refer to move according to the upper path, which has less length and more pheromone.

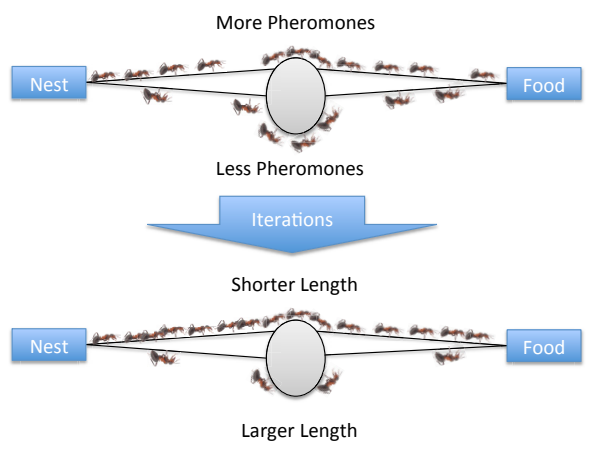

Figure 2. An illustration of Ant Colony Algorithm: ants start from their nest and back when they obtain foods. After a certain iterations, they would like to select the shorter path that areeffected by the more aggregated pheromones. 
The designed intent of ACO is to use for route optimization and many relevant researches show that ACO performs excellent in solving rout optimization problem. However, as mentioned previously, the initial pheromone of the ant colony is merely and leading to a low convergence speed. In addition, the variation of the algorithm is relatively weak. Hence, we embed some operations of GA to refine ACO.

\section{B. Genetic Algorithm}

Genetic Algorithm (GA) is a computational biological evolution model simulating the natural selection and genetic theories of Darwinian. Genetic operation contains three basic genetic operators: selection, crossover and mutation [19]. As detailed in following:

1) Selection: The core idea is to select the winning individuals from the population and eliminate the inferiors. Selection is sometimes called reproduction operator. The purpose is to substitute the better individuals to the next iteration or cross them to generate new individuals. This operation is based on the evaluation of the whole population.

2) Crossover: Genetic recombinant organization plays a central role in the natural process of biological evolution. Similarly, the most important operation of the genetic algorithm is crossover operation. The so-called crossover is to exchange parts of the parents and reconstruct the new individuals. Through this operation, the searching capability has an essential enhancement cause it can let the better parts of two parents get together and form a new individual, which superior to all before.

3) Mutation: The basic content of mutation operator is to change the values of some regions in an individual. It can be regard as an auxiliary for accelerating the crossover operation. It has two goals: first is to enhance the local search capability of GA; another is to maintain the diversity of population. Mutation operation can effectively prevent premature convergence.

\section{Efficient Combination Algorithm}

As mentioned above, artificial intelligence algorithms are frequently introduced to solve WSN route optimization problem. Such as Genetic Algorithm (GA) [10], Ant Colony Optimization (ACO) [9], Simulate Annealing (SA) algorithm [11] and Particle Swarm Optimization (PSO) [12]. Either of them can't achieve the real optimal solution of NP-hard problem. But, every algorithm has its character and advantage.

In order to design an algorithm superior to them, some researchers proposed a certain combination algorithms [23][20][24]. [23] designed PSO-GA and [24] proposed SA-GA. In [20], a GA-ACO model was presented, although they improve the searching capability, but the combination is simple and not focuses on route optimization. These mixed algorithms are better than the original algorithms. From another perspective, we can see that GA can be integrated with other methods stably.

The updating of pheromone in ACO has an explicit advantage for solving route optimization problem. However, because the initialization of pheromone is little and thus the convergence speed is slow. In addition ACO is easy to fall into local optimum and can't jump out. Compared to $\mathrm{ACO}$, although GA is not designed for solving route optimization, but its mutation and crossover operations can be introduced to refine ACO. Either can improve the convergence speed and enhance the solving precision. Thus, we propose two operations to combine the two algorithms and form a novel optimization algorithm for WSN route optimization.

\section{Operation a).}

When an ant is searching for the next node to visit, it often chooses the node that has the highest transformation probability. This leads all ants make the same decisions. Thus the searching capability of ACO declined. In order to keep the diversity of $\mathrm{ACO}$, we add mutation operation in the processing of ants choosing the next node. Let small portion of ants select two nodes and then randomly choose one node.

\section{Operation b).}

After all the ants achieve the sink node, each ant will be regarded as an individual and then processed by the crossover operation, replace the parent ant if the generated new ant is better. Since the crossover operation will generate two new ants with similar routes. This operation will provide the ability to enhance the pheromone near the original route. Thus, searching space has been expanded.

The proposed two operations will significantly enhance the performance of ACO. Parameters analysis will be detailed in Section 4. In order to better exhibit our proposed algorithm, we detailed the flow chart of the combined algorithm in Fig.3

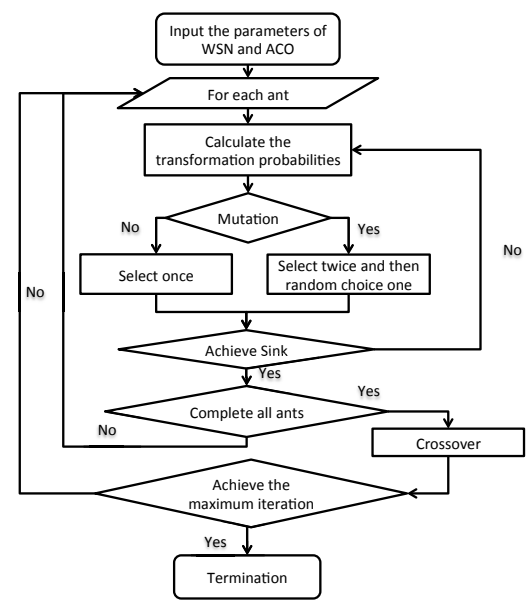

Figure 3. The flowchart of the proposed combining algorithm, which is an effective method for solving WSN route optimization problem.

\section{EXPERIMENTAL RESULTS}

We generate a set of WSN data for constructing experiments with 100 sensor nodes randomly placed in a square area $\left(100 \mathrm{~m}^{*} 100 \mathrm{~m}\right)$. The initial energies of them are same. We use life cycle time as a measure to evaluate different algorithms.

The parameters of ACO are detailed as follows: $m=$ $20, \alpha=0.2, \rho=0.2, \beta=2$, crossover rate $=0.7$, mutation rate $=0.1$, and maximum iteration $=50$. For fair comparisons, the parameters are same in both ACO and GA. The simulation processing is detailed as follows:

Step 1. Initialize the WSN using the above parameters, each node increased by the same amount of energy.

Step 2. Each sensor node finds the optimal route from its location to the Sink node; all the nodes on this path will emit and receive a certain of data. 
Step 3. Terminate the simulation until the energy of any node is consumed completely.

The number of simulations is the network life cycle time.

Before we conduct our simulations, the parameters of the two proposed operations should be analyzed. For the first operation, we suggested that to embed the crossover operation into ACO. However, an excessive proportion of the crossover for the ant colony is not reasonable. It would greatly weaken the meaning of the pheromone and makes the algorithm degenerated into a genetic algorithm. Therefore, we firstly simulate the given WSN and find the optimal ratio of $\operatorname{crossover} p_{1}$.

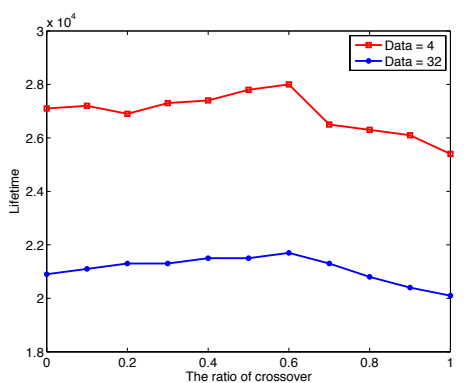

Figure 4. Comparison of results of different ratios of crossover.

As showed in Tab. 1, the optimal $p_{1}=0.6$.Consequently, we analyze another parameter; the selection number of mutation operation $p_{2}$. Obviously, when $p_{2}=0$, the proposed algorithm is equivalent to ACO. However, the algorithm will become a random searching algorithm. The comparison results of different value of $p_{2}$ are showed in Fig.5.

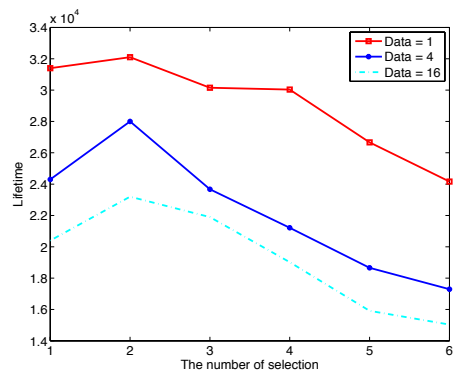

Figure 5. Comparison of results of different selection number.

The life cycle time of WSN is simulated in Fig.6.

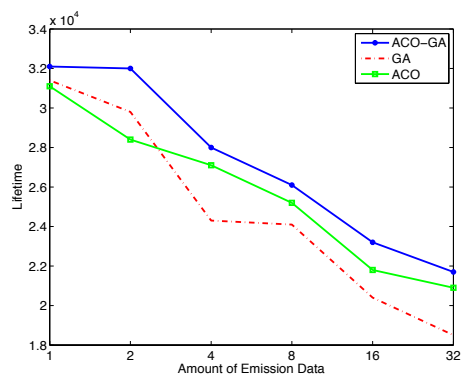

Figure 6. The comparison results on different algorithm.

As can be seen in Fig.6, ACO is slightly better than GA when the case that the emission data are larger. ACOGA has a significant enhancement based on ACO; Fig.7 shows the iterations curves of three methods.

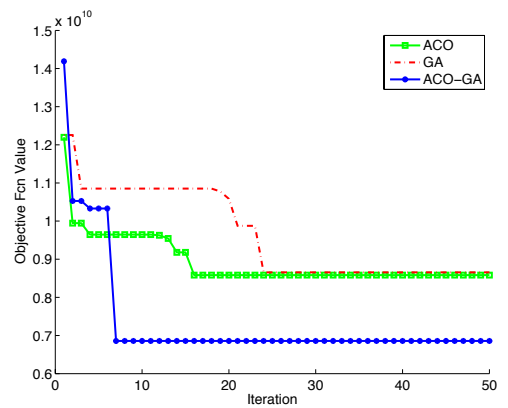

Figure 7. The comparison on the iteration curves of three methods.

Obviously, the proposed ACO-GA is superior to the others; either the coverage speed or optimized results.

Fig. 8 shows the optimized result of a pair of sensor nodes. Obviously, the route that searched by ACO-GA (blue line) is the shortest and the energy consumption is the least cause the transmission using only 9 nodes.

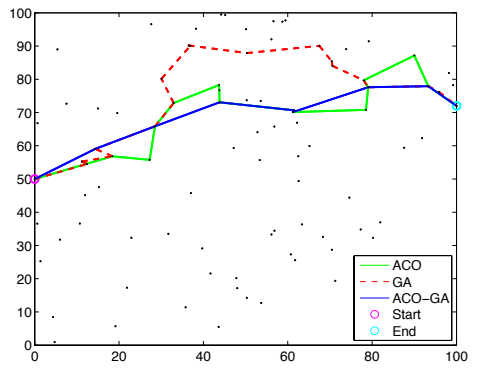

Figure 8. The comparison of the optimized path results by using different algorithms.

\section{CONCLUSION}

This paper focuses on the optimization of WSN route optimization problem. Firstly, we select ACO as the basic tool for solving the optimal route with the shortest length and the least energy consumption. In order to refine the results solving by ACO, GA was embed into ACO and we proposed two operations to form a novel optimization algorithm. Experimental results show that the proposed algorithm achieves the best fitness value.

\section{ACKNOWLEDGMENTS}

The authors wish to thank the Science and Technology Plan Projects of Henan Province for contract 132300410485 and 142300410463 , the Key Research Projects of Universities in Henan Province for contract $15 \mathrm{~A} 520035$ and 15A520124, under which the present work was possible.

\section{REFERENCES}

[1] Ruiz-Garcia L, Lunadei L, Barreiro P, et al. A review of wireless sensor technologies and applications in agriculture and food industry: state of the art and current trends [J]. Sensors, 2009, 9(6): 4728-4750. http://dx.doi.org/10.3390/s90604728

[2] Yick J, Mukherjee B, Ghosal D. Wireless sensor network survey [J]. Computer networks, 2008, 52(12): 2292-2330. http://dx.doi.org/10.1016/j.comnet.2008.04.002

[3] Walters J P, Liang Z, Shi W, et al. Wireless sensor network security: A survey [J]. Security in distributed, grid, mobile, and pervasive computing, 2007, 1: 367 . 
[4] Xiangning F, Yulin S. Improvement on LEACH protocol of wireless sensor network [C]. Sensor Technologies and Applications, 2007. SensorComm 2007. International Conference on. IEEE, 2007: 260-264.

[5] Dietrich I, Dressler F. On the lifetime of wireless sensor networks [J]. ACM Transactions on Sensor Networks (TOSN), 2009, 5(1): 5. http://dx.doi.org/10.1145/1464420.1464425

[6] Anastasi G, Conti M, Di Francesco M, et al. Energy conservation in wireless sensor networks: A survey[J]. Ad hoc networks, 2009, 7(3): 537-568. http://dx.doi.org/10.1016/j.adhoc.2008.06.003

[7] Powell O, Leone P, Rolim J. Energy optimal data propagation in wireless sensor networks [J]. Journal of Parallel and Distributed Computing, 2007, 67(3): 302-317. http://dx.doi.org/10.1016/ j.jpdc.2006.10.007

[8] Zhang J, Li W, Cui D, et al. The NS2-based simulation and research on wireless sensor network route protocol [C]. Wireless Communications, Networking and Mobile Computing, 2009. WiCom'09. 5th International Conference on. IEEE, 2009: 1-4.

[9] Okdem S, Karaboga D. Routing in wireless sensor networks using an ant colony optimization (ACO) router chip [J]. Sensors, 2009, 9(2): 909-921. http://dx.doi.org/10.3390/s90200909

[10] Ferentinos K P, Tsiligiridis T A. Adaptive design optimization of wireless sensor networks using genetic algorithms [J]. Computer Networks, 2007, 51(4): 1031-1051. http://dx.doi.org/10.1016/ j.comnet.2006.06.013

[11] Wang X, Ma J J, Wang S, et al. Distributed particle swarm optimization and simulated annealing for energy-efficient coverage in wireless sensor networks [J]. Sensors, 2007, 7(5): 628-648. http://dx.doi.org/10.3390/s7050628

[12] Kulkarni R V, Venayagamoorthy G K. Particle swarm optimization in wireless-sensor networks: A brief survey [J]. Systems, Man, and Cybernetics, Part C: Applications and Reviews, IEEE Transactions on, 2011, 41(2): 262-267.

[13] Dorigo M, Birattari M. Ant colony optimization $[\mathrm{M}] / /$ Encyclopedia of Machine Learning. Springer US, 2010: 3639.

[14] Lee J W, Lee J J. Ant-colony-based scheduling algorithm for energy-efficient coverage of WSN [J]. Sensors Journal, IEEE, 2012, 12(10): 3036-3046. http://dx.doi.org/10.1109/JSEN. 2012.2208742

[15] Yu B, Yang Z Z, Yao B. An improved ant colony optimization for vehicle routing problem [J]. European journal of operational research, 2009, 196(1): 171-176. http://dx.doi.org/10.1016/ j.ejor.2008.02.028

[16] Han Y, Shi P. An improved ant colony algorithm for fuzzy clustering in image segmentation [J]. Neurocomputing, 2007, 70(4): 665671. http://dx.doi.org/10.1016/j.neucom.2006.10.022

[17] Panda S, Padhy N P. Comparison of particle swarm optimization and genetic algorithm for FACTS-based controller design [J]. Applied soft computing, 2008, 8(4): 1418-1427. http://dx.doi.org/10.1016/j.asoc.2007.10.009

[18] Toğan V, Daloğlu A T. An improved genetic algorithm with initial population strategy and self-adaptive member grouping $[\mathrm{J}]$. Computers \& Structures, 2008, 86(11): 1204-1218.

[19] Cantú-Paz E. A survey of parallel genetic algorithms [J]. Calculateurs paralleles, reseaux et systems repartis, 1998, 10(2): 141171.

[20] Lee Z J, Su S F, Chuang C C, et al. Genetic algorithm with ant colony optimization (GA-ACO) for multiple sequence alignment
[J]. Applied Soft Computing, 2008, 8(1): 55-78. http://dx.doi.org/10.1016/j.asoc.2006.10.012

[21] Hasenfratz D, Meier A, Moser C, et al. Analysis, comparison, and optimization of routing protocols for energy harvesting wireless sensor networks $[\mathrm{C}]$. Sensor Networks, Ubiquitous, and Trustworthy Computing (SUTC), 2010 IEEE International Conference on. IEEE, 2010: 19-26.

[22] Luo J, Hubaux J P. Joint sink mobility and routing to maximize the lifetime of wireless sensor networks: the case of constrained mobility [J]. IEEE/ACM Transactions on Networking (TON), 2010, 18(3): 871-884. http://dx.doi.org/10.1109/TNET.2009. 2033472

[23] Robinson J, Sinton S, Rahmat-Samii Y. Particle swarm, genetic algorithm, and their hybrids: optimization of a profiled corrugated horn antenna[C]. Antennas and Propagation Society International Symposium, 2002. IEEE. IEEE, 2002, 1: 314-317. http://dx.doi.org/10.1109/aps.2002.1016311

[24] Leung T W, Chan C K, Troutt M D. Application of a mixed simulated annealing-genetic algorithm heuristic for the twodimensional orthogonal packing problem [J]. European Journal of Operational Research, 2003, 145(3): 530-542. http://dx.doi.org/10.1016/S0377-2217(02)00218-7

\section{AUTHORS}

Yongfeng Cui received the BS degree in Computer Science and Technology from Henan Normal University and the MS degree in Computer Application Technology from Huazhong University of Science and Technology, China in 2000 and 2007 respectively. He is currently researching on Computer Application Technology (CAT). $\mathrm{He}$ is with is with the School of Science and Technology, Zhoukou Normal University, Zhoukou, Henan, 466001, China (e-mail: cuiyf@zknu.edu.cn).

Wei Liu received the BS degree in computer science and technology from Henan Normal University and the MS degree in Computer Technology from The PLA Information Engineering University, China in 1996 and 2006 respectively. He is currently researching on Network Technology (NT) and Computer Application Technology (CAT). He is with School of Science and Technology, Zhoukou Normal University, Zhoukou, Henan, 466001, China (e-mail: liuwei@zknu.edu.cn).

Zhongyuan Zhao, engineering graduate student, Henan University of Technology. Received the BS degree in computer science and technology from Zhoukou Normal University, China in 2009. He is currently researching on Computer Application Technology (CAT). He is with the School of Science and Technology, Zhoukou Normal University, Zhoukou, Henan, 466001, China and the College of Information Science and Engineering, Henan University of Technology, Zhengzhou, Henan 450052, China (e-mail: zhaozy@zknu.edu.cn).

Submitted 21 September 2015. Published as resubmitted by the authors 20 October 2015. 